\title{
Expression of Cripto-1 in the placenta and its role in placenta accreta and placenta previa
}

\author{
Lingling Jiang ${ }^{1}$, Xiaoya $\mathrm{Wu}^{2}$, Jianying Yan ${ }^{1 *}$, Rongxin Chen ${ }^{1}$, Qing Han ${ }^{1}$, Qinjian Zhang ${ }^{1}$ \\ ${ }^{1}$ Fujian Provincial Maternity and Children's Hospital, affiliated hospital of Fujian Medical University, Fuzhou, Fujian, China \\ ${ }^{2}$ Fujian Provincial Hospital, affiliated hospital of Fujian Medical University, Fuzhou, Fujian, China \\ ${ }^{*}$ Co-first author: Jianying Yan
}

\begin{abstract}
Objectives: This study Aims to explore the role of placental Cripto-1 in the incidence of an adherent placenta.

Material and methods: Ten pregnant women with placenta increta, 20 pregnant women with placenta previa and 30 women with normal pregnant were enrolled in this study. Reverse transcription-polymerase chain reaction (RT-PCR) was used to measure the expression of Cripto-1 in the placenta while as the analysis of placental Cripto-1 was performed by Western blotting

Results: The placenta increta group showed higher levels of Cripto- 1 in the center of the increta as compared to the non-implantation area. The level of placental Cripto-1 in the placenta increta was higher than that of the placenta accrete. The expression of placental Cripto-1 in the placenta increta and placenta previa groups was higher than that of control.

Conclusions: Placental Cripto- 1 is involved in the regulation of placental tissue invasion. Additionally, excessive placental growth or penetration into the myometrium are likely to be involved in the development of placenta increta.
\end{abstract}

Key words: Cripto-1; placenta increta; placenta previa; pathogenesis; pregnancy

Ginekologia Polska 2019; 90, 2: 86-92

\section{INTRODUCTION}

Placenta increta $(\mathrm{PA})$ refers to the chorionic villi invasion of the myometrium caused by an abnormal placenta. It can be divided into three categories: adhesive placenta, placenta accrete and placenta percreta, according to the depth of the invasion. Placenta increta, also referred to as pathological placenta adhesion [1], is a serious complication in the field of obstetrics. Recent researches on placenta implantation especially those exploring risk factors, diagnosis, treatment methods, and maternal and fetal outcome are lacking. To date, there are few studies on the etiology and pathogenesis of placental implantation. The mechanisms responsible for placenta accreta are not completely understood. Although it is generally accepted that decidual dysplasia, trophoblast cell invasion ability enhancement, and vascular remodeling may be of importance in elucidating the pathophysiology of placenta accreta [2], the mechanisms responsible for its occurrence are still not completely understood. Cripto- 1 is a glycosylphosphatidylinositol-anchored small molecular signaling protein and a member of epidermal growth fac- tors EGF-CFC family. In early embryonic development, Cripto-1 plays an important role in germ layer differentiation and later in each organ development. It plays a role in the activation of multiple signaling pathways regulating tumor cell proliferation, differentiation, and migration. Placental trophoblast cells and tumor cells have similar biological characteristics, and Cripto- 1 can control their migration and invasion as well. So, we assume that the effect of placental trophoblast cells on cell morphology and their ability to promote placenta implantation might be an important factor in the pathogenesis of placenta increta. Higher expression of Cripto-1 can lead to an increase in cell proliferation, migration and invasion, abnormal placenta angiogenesis, enhancement of placental trophoblast cell invasive ability, and placental invasion. The present study examined the levels of Cripto-1 in pregnant women with placenta accreta.

\section{MATERIAL AND METHODS}

Enrolled subjects were pregnant women with regular antenatal care and hospitalized for cesarean section at 
the Obstetric Department of Fujian Provincial Maternity and Children's Hospital from January to December 2015. All subjects were Chinese nationals. Based on clinical manifestations, ultrasonic testing, and pathological examination, 10 cases were identified as placenta accreta (PA) and 20 as placenta previa (PP). An additional thirty pregnant women who received cesarean section due to a scarred uterus, abnormal fetal position, abnormal obstetric canal, and social factors were selected to form the control group (NC). This study referred to the relevant literature [3] for the diagnostic criteria of PP and PA. Late pregnancy is defined as 28 weeks or later. Those with both PP and PA were included in the PA group. No included subject underwent labor and nor had a premature rupture of membranes at the time of cesarean section. None of the women were in active labor, had rupture of fetal membranes, or had clinical signs of infection. Patients with pregnancy complications and surgical complications were excluded from the study. All subjects had a single pregnancy. Informed consents were obtained from each subject, and the protocol for this study was approved by the local Institutional Review Board (Ethics committee of Fujian provincial maternal and child health hospital, 20140928).

\section{Specimen collection}

Immediately after cesarean section, the placental tissues were taken aseptically from the maternal surface of the placenta. Specific sites of sample collection in each group are described as follows: samples were collected from an accreta area and a none-accreta area for the PA group whereas for the PP group, placental tissues were collected from a marginal location and a central location. For the normal group, placental tissues were only collected from the central area. Tissue samples were incised at $1.0 \times 1.0 \times 1.0 \mathrm{~cm}$ under sterile conditions. Sites with hemorrhage, necrosis, and calcification were avoided. After rinsing with cold saline, placental samples were immediately put into a $1.5 \mathrm{~mL}$ EP tube, frozen in liquid nitrogen and subsequently transferred into sterile tubes for storage at $-70^{\circ} \mathrm{C}$ until assayed. Repeated freezing and thawing were avoided.

\section{Main materials}

The real-time fluorescence quantification PCR assay was purchased from Applied Biosystems (ABI), while nucleic acid and protein quantitative determination apparatus, and low-temperature high-speed centrifugal machine were from Thermo. The horizontal nucleic acid electrophoresis apparatus was from the Beijing Liuyi Biotechnology Co., Ltd. The gel-imaging scanner was from BIO-Rad, USA. The rabbit anti-human Cripto-1 monoclonal antibody was purchased from Abcam. The rabbit anti-human GAPDH polyclonal antibody, horseradish peroxidase-conjugated anti-rabbit secondary antibody, and high-sensitivity chemiluminescence assay kit were from Kangwei Shiji Biotechnology Company, Beijing. The PCR primers were from Beijing Dingguo Changsheng Biotechnology company and SYBR Green I (10x) was from Genview.

\section{Immunohistochemistry}

After dehydration and paraffin embedding, tissues were sliced into $3 \mu \mathrm{m}$ thick sections and adsorbed on adhesion slides for hot repair $\left(88^{\circ} \mathrm{C}\right.$ for $\left.10 \mathrm{~min}\right)$. The slides were dewaxed, hydrated with graded ethanol and immersed in sodium citrate solution under high-temperature conditions for 1-2 min for antigen repair. After cooling, the slides were washed with Tris-buffered saline (TBS). A 30\% hydrogen peroxide solution was used to block endogenous peroxidase for $10 \mathrm{~min}$ followed by incubation with sheep serum for $30 \mathrm{~min}$ at room temperature and binding with the nonspecific antibody. Monoclonal mouse anti-human antibodies against Cripto-1 (Abcam, USA) were added to the slides at a dilution of 1:100 and incubated at $4^{\circ} \mathrm{C}$ overnight. Biotinylated rabbit anti-mouse antibody (Abcam, USA) was used at a dilution of 1:100 for $30 \mathrm{~min}$ at room temperature. After washing with TBS, the specimens were stained with diaminobenzidine, and hematoxylin and coverslipped for microscopic observation. Phosphate buffer solution instead of the primary antibody was used as a negative control.

\section{Western blot}

Tissue samples were first washed with PBS three times then washed with lymphocyte lysis buffer. Proteins were purified with the addition of the extraction buffer. Protein concentration was measured by the BCA assay. According to the results of the protein quantification, the corresponding volume of total protein and $5 x$ protein gel electrophoresis buffer were mixed and the protein denatured for $10 \mathrm{~min}$ at $95^{\circ} \mathrm{C}$. The gel was pre-electrophoresed for $10 \mathrm{~min}$ at a constant $80 \mathrm{~V}$ until the leading edge of the bromophenol blue reached the separation gel. The voltage was adjusted to provide a constant $120 \mathrm{~V}$ until the dye reached the bottom of the separation gel. The protein was transferred at a constant $110 \mathrm{~V}$ for $1 \mathrm{~h}$. Membranes were blocked for two hours at room temperature in Tris-buffered saline-Tween-20 (TBS-T) containing $10 \%$ skimmed milk. Membranes were then incubated overnight with the appropriate primary antibody (a rabbit monoclonal antibody against Cripto-1, Abcam Company, UK) diluted in TBS-T with $3 \%$ BSA at $4^{\circ} \mathrm{C}$. Membranes were then incubated with secondary antibodies (an anti-rabbit lgG antibody, Beijing Dingguo Changsheng Biotechnology Company, China) for one hour at room temperature after washing with TBS-T. Blots were washed three times with TBS-T, and the detection was performed using the BM Chemiluminescence system. After exposure, membranes were stained with Amido Black Staining Solution to calculate variations in protein content among samples. Densitometric analysis of band 


\begin{tabular}{|l|l|l|l|l|l|l|}
\multicolumn{6}{|l|}{ Table 1. Comparison of the clinical situations of the three groups of pregnant women } \\
\hline Group & No. of Cases & Age (Y) & $\begin{array}{l}\text { Gestational Weeks } \\
\text { (Week) }\end{array}$ & $\begin{array}{l}\text { Number of Pregnancies } \\
\text { (Times) }\end{array}$ & $\begin{array}{l}\text { Number of Uterine } \\
\text { Surgeries (Times) }\end{array}$ & Hospital Stay (Day) \\
\hline PA & 10 & $31.7 \pm 6.6$ & $35.3 \pm 4.0^{*}$ & $2.7 \pm 1.8^{*}$ & $0.7 \pm 0.7$ & $8.0 \pm 4.3^{*}$ \\
\hline PP & 20 & $30.3 \pm 5.8$ & $36.2 \pm 2.5^{*}$ & $1.5 \pm 1.5$ & $0.4 \pm 0.8$ & $6.5 \pm 2.9$ \\
\hline CON & 30 & $30.2 \pm 3.8$ & $39.2 \pm 1.0$ & $1.0 \pm 0.8$ & $0.5 \pm 0.6$ & $5.4 \pm 1.7$ \\
\hline
\end{tabular}

* $P<0.05$

Table 2. Comparison of the clinical situations of the three groups of pregnant women

\begin{tabular}{|l|l|l|l|l|l|}
\hline Group & $\begin{array}{l}\text { Duration of } \\
\text { Operation (Hour) }\end{array}$ & $\begin{array}{l}\text { Intraoperative } \\
\text { Hemorrhage (ML) }\end{array}$ & Placental Weight (G) & Weight of Newborn (G) & $\begin{array}{l}\text { Hospitalization Costs } \\
\text { (in RMB 10,000) }\end{array}$ \\
\hline PA & $1.6 \pm 1.0^{*}$ & $1280.0 \pm 1316.9^{*}$ & $511.0 \pm 147.8^{*}$ & $2441 \pm 913.7^{*}$ & $2.2 \pm 1.6^{*}$ \\
\hline PP & $0.9 \pm 0.3$ & $543 \pm 179.7$ & $577.8 \pm 106.5^{*}$ & $2651.1 \pm 644.7^{*}$ & $1.2 \pm 0.3$ \\
\hline CON & $0.9 \pm 0.3$ & $383.3 \pm 130.9$ & $673.6 \pm 147.3$ & $3368.8 \pm 528.6$ & $0.9 \pm 0.1$ \\
\hline
\end{tabular}

* $\mathrm{P}<0.05$

intensities was made with Basic Quantifier software. $\beta$-actin was used as a loading control. We compared the gray values of the Cripto- 1 and $\beta$-actin protein bands.

\section{RTQ-PCR}

Trizol was used to extract total RNA from placental tissues. A spectrophotometer was used to measure the light absorption values (A), and total RNA concentration and purity were calculated from $260 \mathrm{~nm}$ and $280 \mathrm{n}$ absorption. RNA integrity was determined by $1 \%$ agarose gel electrophoresis. A sample of $2 \mathrm{ug}$ of total RNA was reverse transcribed into cDNA. Real-time fluorescence quantification PCR was used for quantitative determination. The full-length sequence of the target gene mRNA was obtained from GenBank. Primer 5.0 was used to design the primer sequence. After Blast analysis, the primer sequence was shown to have the necessary specificity. This study entrusted Beijing Dingguo Changsheng Biotechnology Co., Ltd. to synthesize all the primers. $\beta$-actin: The upstream primer is $5^{\prime}$-ATC ATG TTT GAG ACC TTC AAC A-3', while the downstream primer is 5'-CAT CTCTTG CTG AAG ECC A-3'. Cripto-1:The upstream primer is

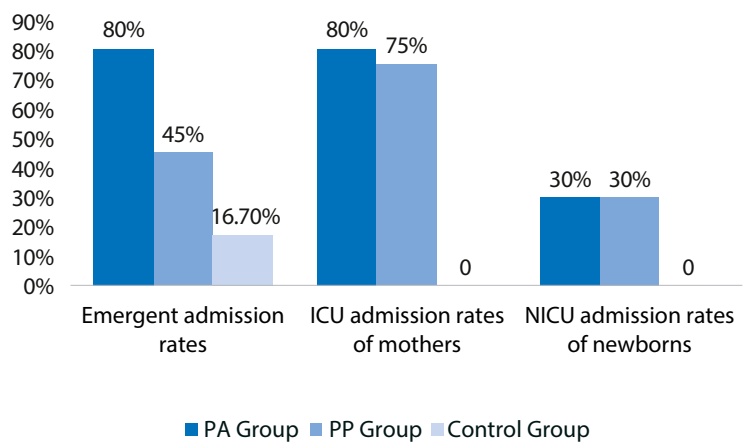

Figure 1. Analysis of hospitalization
5'-CAG GGA GACTGG GTA GGA A-3', while the downstream primer is 5'- TGT TGG GGA CAT TGA GGT A-3'. The RT-PCR reaction conditions and procedures were performed according to the instructions, and $2^{-\triangle \Delta C T}$ was used to conduct a relative quantitative analysis of the results.

\section{Statistical analysis}

Statistical analysis was carried out with the Statistical Package for Social Science Software (SPSS19.0). Data are presented as mean $\pm \mathrm{SD}$. Differences among groups were compared with one-way ANOVA. Comparison between two groups was performed with t-test or Wilcoxon and Kruskal Wallis tests, while comparison among the three groups was performed with one-way ANOVA. A P-value $<0.05$ was regarded as statistically significant.

\section{RESULTS}

\section{Baseline characteristics of subjects from the three groups}

The differences in age and number of cesarean sections between patients in the groups were not statistically significant. The number of pregnancies, hospital stays, duration of operation, intraoperative hemorrhage, and hospitalization costs of the PA group were significantly higher than those of the Control group, $(P<0.05)$. Gestational age, the placenta weight, and weight of the infant in both the PA and PP groups were significantly lower than those of the Control group $(P<0.05)($ Tab. 1, 2).

The emergency admission rates of the PA group, PP group, and the Control group were $80 \%, 45 \%$, and $16.7 \%$, respectively. Meanwhile, the ICU admission rates of the mothers were $80 \%$ for the PA group, $75 \%$ for the PP group and $0 \%$ for the Control group. The NICU admission rates of newborns were $30 \%, 30 \%$, and $0 \%$, respectively. (Fig. 1). 


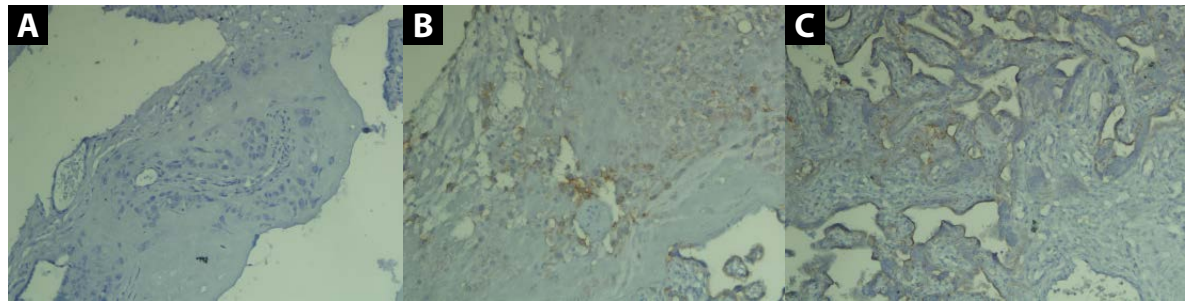

Figure 2. Expression and distribution of Cripto-1 in placental tissue

A - negative control (100x); B - showed the expression of Cripto-1 in placenta tissues in group PA (100x); C - showed the expression of Cripto-1 in placenta tissues in group PA (100x). It was positive for cytoplasmic brown staining
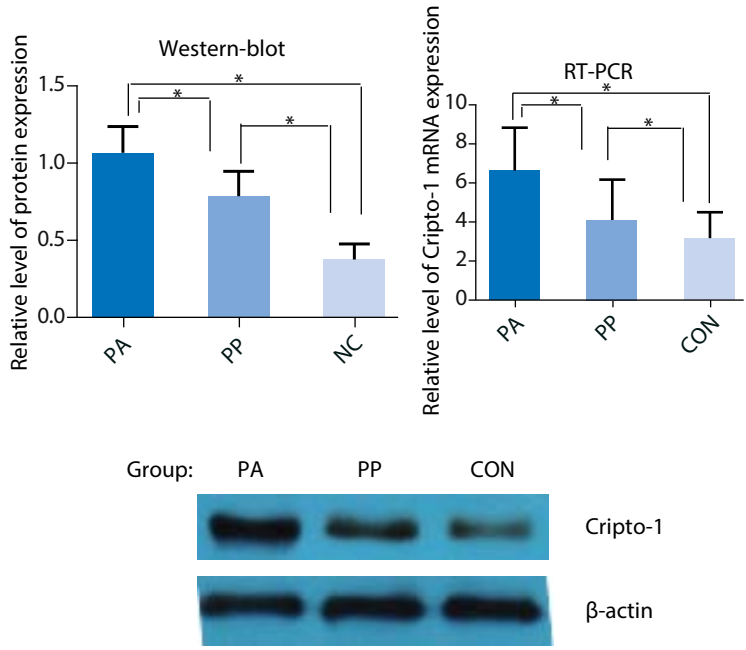

Cripto-1

$\beta$-actin

Figure 3. Cripto-1 expression of the pregnant women of the three groups

\section{Cripto-1 expression levels in placental tissues of the three groups \\ Localization of Cripto-1 in the placenta}

Immunohistochemistry indicated the presence of Cripto-1 in placental tissues of subjects from the three groups. The expressions of Cripto-1 in placental tissues of PA and PP groups were higher than that of the control group. Positive staining showed brown yellow granules within the tissue (Fig. 2).

\section{Cripto-1 expression levels in placental tissues of the three groups}

The ratios of Cripto- 1 protein gray value/ $\beta$-actin protein gray value of the three groups were:PA group $1.054 \pm 0.178$, PP group $0.774 \pm 0.170$, and the Control group $0.369 \pm 0.110$. The relative content of Cripto-1 protein in the PA and PP groups were significantly higher than that of the Control group $(P<0.05)$. The Cripto-1 protein level in the PA group was much higher than that of PP group $(P<0.05)$. (Fig. 3).

\section{Cripto-1 expression at different sites in the PA group}

There was a significant difference in average levels of Cripto- 1 protein between samples from the accreta area $1.206 \pm 0.038$ and the none-accreta area $0.901 \pm 0.119$ in the
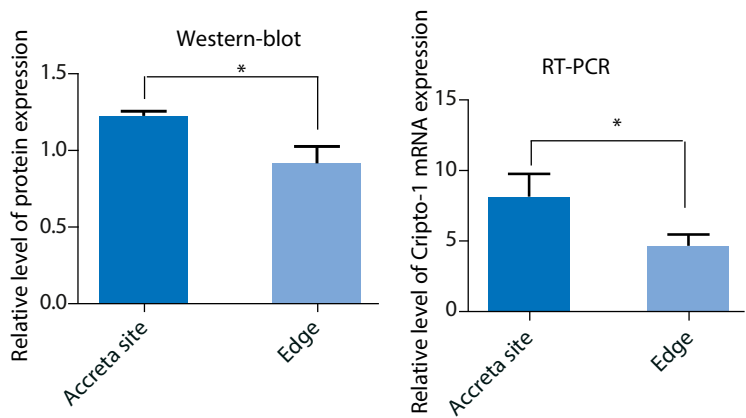

Figure 4. Cripto-1 expression of different sites of PA Group

PA group. The relative content of Cripto-1 mRNA in the PA group was $7.971 \pm 1.751$ at the central area and $4.520 \pm 0.852$ at the none-accreta area. The difference between the two sites was statistically significant $(P<0.05)$. (Fig. 4).

\section{Cripto-1 expression at different sites in the PP group and the Control group}

The relative content of Cripto- 1 protein in the PP group was $0.739 \pm 0.136$ at the central area and $0.810 \pm 0.196$ at the edge. The difference between the two sites was not statistically significant. No significant difference in the relative content of Cripto- 1 protein was seen between the central area $(0.368 \pm 0.112)$ and the edge $(0.370 \pm 0.110)$ in the Control group.

The relative content of Cripto-1 mRNA in the PP group was $3.680 \pm 2.227$ at the central area and $4.262 \pm 2.227$ at the edge. The difference between the two sites was not statistically significant. The relative content of Cripto- 1 mRNA in the Control group was $3.045 \pm 1.447$ at the central area and $3.030 \pm 1.411$ at the edge. The difference between the two sites was not statistically significant. (Fig. 5).

\section{Cripto-1 expression in different types of accreta}

In the PA group, the Cripto-1 expression levels were significantly higher in the placenta increta (PI) tissues than in the placenta accreta $(P<0.05)$. (Tab. 3, Fig. 6).

\section{DISCUSSION}

Placenta accreta is a serious obstetric complication. The recent years have seen a sharp increase in its incidence with the increase in uterine surgeries like cesarean section and 


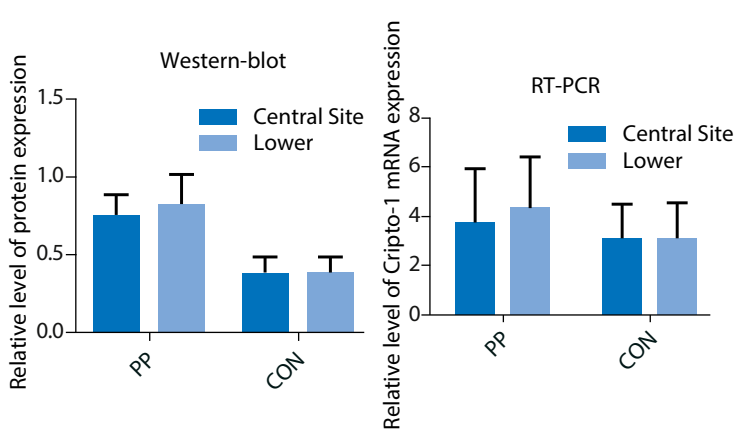

Figure 5. Cripto-1 expression of different sites of PP Group and the Control Group

Table 3. Cripto-1 expression of different accreta types

\begin{tabular}{|l|l|l|}
\hline Group & Western-blot & RT-PCR \\
\hline Placenta increta $(n=8)$ & $1.071 \pm 0.219$ & $6.676 \pm 2.224$ \\
\hline Placenta accreta $(n=2)$ & $0.984 \pm 0.170$ & $4.523 \pm 1.250$ \\
\hline
\end{tabular}
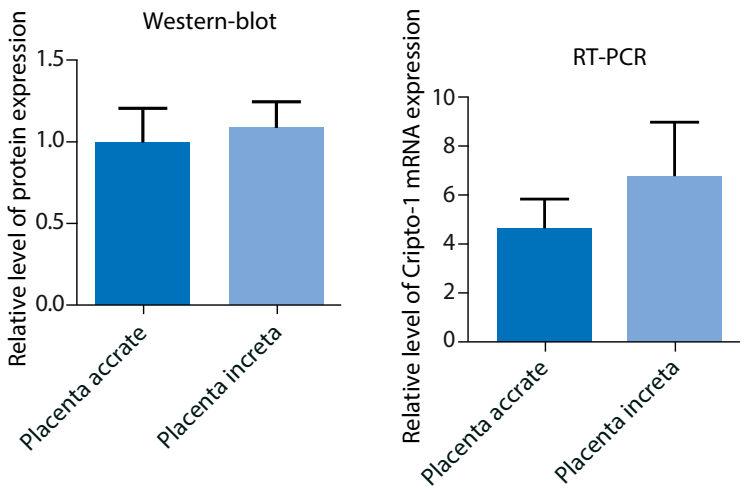

Figure 6. Cripto-1 expression of different accreta

abortion. China reports that the morbidity from placental accreta has reached $0.4 \%$ domestically [4]. Its clinical manifestations include spontaneous uterine perforation during pregnancy and no, or partial, separation of the placenta during delivery. These can lead to life-threatening complications such as hemorrhage, shock, secondary infection, or even death during childbirth. It is reported that the rate of hysterectomy caused by PA has reached $77.8 \%$ currently making PA a leading cause of hysterectomy due to massive bleeding [5]. The mortality rate of pregnant women due to PA has reached $7 \%$. The clinical data of this study indicate that the gestational age at delivery in the PA group is lower than that of the Control group. Additionally, the length of hospitalization, the duration of operation, the occurrence of intraoperative hemorrhage, hospitalization costs, emergency admission rate, and ICU admission rate are also higher in the PA group. Newborns in the PA group have been shown to have a lower birth weight, a higher NICU admission rate, and premature birth rate. Comparison within the emergency admission cases has shown that planned delivery can reduce the amount of bleeding, the incidence of other complications, and the length of an ICU stay. PA not only represents a serious health threat to mother and child but also increases the costs of medical services and is a great economic burden on families.

At present, the etiology of PA is still not fully understood, and multiple factors are implicated in its pathogenesis. It is of tremendous significance to be able to clarify its pathogenesis, develop early detection means and interventional methods to avoid its complications. Recent research has indicated that decidual maldevelopment, enhanced trophoblast invasion, and abnormal vascular remodeling may be important causes [6].

The results of this study found that, compared with the Control group, the PA group had higher expressions levels of Cripto-1. Moreover, the expression levels varied by anatomic sites. Cripto- 1 expression level at the central area is much higher than that in the none-accreta area. This is indicative of enhancement of trophoblast invasion and excessive placental invasion. The PP group also showed higher levels of Cripto-1 expression when compared to the Control group. However, the difference between Cripto- 1 expressions in the central site and the edge in the PP group is not statistically significant. Excessive trophoblast invasion was not observed in the PP group even though Cripto-1 expressions varied according to the sampling site. This might be due to lower Cripto-1 expressions in the PP group as compared to the PA group. The cell signal intensity was low and did not reach the threshold of excessive trophoblast invasion in the PP group. There is also the possibility that the up-regulation of Cripto-1 expression is only one of the conditions for the enhancement of trophoblast invasion. There are other vital factors to be explored that might affect excessive trophoblast invasion. Only two cases of placenta accreta were collected in this study. Western-blot results indicated that Cripto-1 expression levels in placental tissues are significantly higher in subjects with placenta increta than those with placenta accreta.

During normal pregnancy, trophoblast cells shift from epithelial phenotype to mesenchymal phenotype via EMT and differentiate into extravillous trophoblast cells with strong invasive ability. The EVTS migrate to the uterine decidua, infiltrating along the maternal spiral artery in a retrograde direction, gradually replacing vascular endothelial cells, and transforming into non-invasive cell phenotypes like endothelial trophoblasts and myometrial multinuclear giant cells via MET. During a normal pregnancy, the invasive ability of trophoblast cells is confined within a certain time and space. Therefore, the invasion is limited in depth. 
The Cripto-1 gene was first found and separated by Ciccodicola et al. in the human teratoma NTERA2/D1 cell line cDNA library in 1989 [7]. Cripto-1 is expressed at a higher level during early embryonic development but is weakly expressed or not detected in the placenta in the second and the third trimesters of normal pregnancy. The up-regulation of Cripto-1 may destroy normal EMT and MET regulatory mechanisms of trophoblast cells and activate abnormal signal pathways to strengthen the duration and intensity of trophoblast invasion.

Consistent with other studies, the results of this study indicate that the expression levels of Cripto-1 protein and mRNA in both the PA and PP groups are higher than in the Control group. Bandeira et al. [8] adopted an immunohistochemical technique to determine the Cripto-1 expression in the placenta during the third trimester of pregnancy. They have reported that Cripto-1 expression in the PA group was significantly higher than in the Control group and that the expression level increases along with the depth of accreta. Additionally, they have put forward for the first time the idea that EVT is the main cell expressing Cripto- 1 and that With the morphological changes of EVT at the site of accreta, trophoblast cells clustered together clone-like or were dispersedly distributed. They have exhibited a greater cell volume with scattered cells showing migrational characteristics and a star-shaped cytoplasm or the presence of long-axis protrusions. The number of multinucleated giant cells in the myometrium was significantly reduced. Kim et al. [9] found that the thickness of the ETV cell layer in the accreta area was significantly higher than that of a normal placenta. In the third trimester of normal pregnancy, EVT cells showed no proliferative activity and demonstrated a low apoptotic index. Moreover, the differentiation of many multinuclear giant cells from trophoblast cells at their final stage was detected. Trophoblast cells invading the myometrium and expressing high levels of Cripto-1 in EVT is consistent with Cripto-1 promoting the migration and invasion of trophoblast cells.

A high-level of expression of Cripto-1 may mediate several mechanisms thus enhancing the invasive ability of trophoblast cells and participating in the pathogenesis of placenta accreta. The increased expression of Cripto- 1 in trophoblast cells may interact with the ability of the TGF- $\beta$ family to regulate classic EMT transcription factors. The transcription and translation of Snail, Twist, and Slug will promote EMT [10]. In contrast, for PA, the EMT of trophoblast cells does not develop toward tumor formation. There may be a complex regulatory network that limits EMT within a controllable range. The wnt/ $\beta$-catenin signaling pathway plays a crucial role in promoting the migration and invasion of trophoblast cells. In the Cripto-1 gene promoter area, there is a binding area for T-cell factor/lymphoid enhancer factor (Tcf/Lef) which is regulated by the Wnt/ $\beta$-catenin sig- nal pathway. Up-regulated Cripto-1 can also serve as a Wnt 11 co-receptor in the cell membrane and, together with Glypican-4 and Frizzled 7, activate $\beta$-catenin. The $\beta$-catenin in the cytoplasm can enter into the nucleus and, as a coactivator of specific DNA-binding protein transcription in the nucleus, upregulate Cripto- 1 expression but also regulate the expression of c-Myc and cyclin D1 (factors related to cell adhesion and cell morphological changes). The migration and invasion of the EVTS are enhanced through a complex cell regulatory network and interaction with expressed factors. Previous studies have found that E-cadherin participates in placenta accreta by regulating trophoblast invasion [11]. Cripto-1 activates the $\mathrm{Wnt} / \beta$-catenin signal pathway, reduces free $\beta$-catenin in the cytoplasm, affects the interaction between $\beta$-catenin and $E$-cadherin as well as the number and stability of cadherin-catenin complexes, a connecting structure of cell adhesion, and increases cell migration and invasion.

Cripto-1 promotes the differentiation of HUVECs into vascular-like structures in vitro and increases the microvessel density of MCF-7 tumors in nude mice [12]. Furthermore, by regulating a specific signaling pathway, it can promote vascular remodeling and neovascularization in PA. Members of the Cripto family can interact with glucose regulatory protein 78, promoting the activation of both TGF-beta and Src/MAPK/PI3K signaling pathways. Its downstream signaling molecules TGF-beta, a bone morphogenetic protein, and Akt are involved in the regulation of natural killer cells and Sertoli cell differentiation [13]. Natural killer cells infiltrate the decidual tissue and promote abnormal vascular remodeling by regulating trophoblast cells which in turn invade the uterus to replace the endothelial cells and the membrane integrity of the uterine spiral artery [14]. MMPs also play a crucial role in vascular remodeling. Cripto-1 promotes MMP2 production via the TGF-beta body/Cripto-1/Smad2 signaling pathway [15]. Cripto-1 participation in placenta increta may be through vascular remodeling.

\section{CONCLUSIONS}

In summary, the pathogenesis of placenta accreta may include decidua maldevelopment, enhanced trophoblast invasion, and abnormal vascular remodeling. Upregulation of Cripto-1 expression strengthens trophoblast invasion. Excessive placental invasion may penetrate the myometrium and lead to the development of placenta accreta. Therefore, the role of Cripto- 1 in the promotion of vascularization during the development of placenta accreta is worth being further explored.

\section{Acknowledgments}

This work was supported by the Key Clinical Specialty Discipline Construction Fujian, P.R.C. (2015 No. 593). And thanks to the people who helped me during specimen collection. 


\section{Conflicts of interest}

All authors declared no potential conflicts of interest related to the authorship and publication of this article.

\section{REFERENCES}

1. Lim BH, Palacios-Jaraquemada JM. The morbidly adherent placenta--a continuing diagnostic and management challenge. BJOG. 2015; 122(12): 1673, doi: 10.1111/1471-0528.13561, indexed in Pubmed: 26498304.

2. Garmi G, Salim R. Epidemiology, etiology, diagnosis, and management of placenta accreta. Obstet Gynecol Int. 2012; 2012: 873929, doi: 10.1155/2012/873929, indexed in Pubmed: 22645616.

3. Leveno KJ, Cunningham FG, Norman FG, et al. Williams Manual of Obstetrics, 24 st edition. McGraw-Hill Education - Europe .

4. Dunjin C, Chunhong S. Placenta Accreta.Chunhong S. ed. Hunan Science and Technology Press, Changsha : 1-6.

5. Sakinci M, Kuru O, Tosun M, et al. Clinical analysis of emergency peripartum hysterectomies in a tertiary center. Clin Exp Obstet Gynecol. 2014; 41(6): 654-658, indexed in Pubmed: 25551957.

6. Parra-Herran C, Djordjevic B. Histopathology of Placenta Creta: Chorionic Villi Intrusion into Myometrial Vascular Spaces and Extravillous Trophoblast Proliferation are Frequent and Specific Findings With Implications for Diagnosis and Pathogenesis. Int J Gynecol Pathol. 2016; 35(6): 497-508, doi: 10.1097/PGP.0000000000000250, indexed in Pubmed: 26630223.

7. Ciccodicola A, Dono R, Obici S, et al. Molecular characterization of a gene of the'EGF family' expressed in undifferentiated human NTERA2 teratocarcinoma cells. EMBO J. 1989; 8(7): 1987-1991, indexed in Pubmed: 2792079

8. Bandeira $\mathrm{CL}$, Urban Borbely A, Pulcineli Vieira Francisco $\mathrm{R}$, et al Tumorigenic factor CRIPTO-1 is immunolocalized in extravillous cytotrophoblast in placenta creta. Biomed Res Int. 2014; 2014: 892856, doi: 10.1155/2014/892856, indexed in Pubmed: 25165718.

9. Kim KR, Jun SY, Kim JY, et al. Implantation site intermediate trophoblasts in placenta cretas. Mod Pathol. 2004; 17(12): 1483-1490, doi: 10.1038/modpathol.3800210, indexed in Pubmed: 15205687.

10. Wendt MK, Allington TM, Schiemann WP. Mechanisms of the epithelial-mesenchymal transition by TGF-beta. Future Oncol. 2009; 5(8): 1145-1168, doi: 10.2217/fon.09.90, indexed in Pubmed: 19852727.

11. Qinjian Z, Jianying Y. Role of E-cadherin in Placenta Accreta [J]. Chinese Journal of Perinatal Medicine. 2015; 4: 306-309.

12. Bianco C, Strizzi L, Ebert A, et al. Role of human cripto-1 in tumor angiogenesis. J Natl Cancer Inst. 2005; 97(2): 132-141, doi: 10.1093/jnci/dji011, indexed in Pubmed: 15657343.

13. Gray PC, Vale W. Cripto/GRP78 modulation of the TGF- $\beta$ pathway in development and oncogenesis. FEBS Lett. 2012; 586(14): 1836-1845, doi: 10.1016/j.febslet.2012.01.051, indexed in Pubmed: 22306319.

14. Wallace $A E$, Fraser R, Cartwright JE. Extravillous trophoblast and decidual natural killer cells: a remodelling partnership. Hum Reprod Update. 2012; 18(4): 458-471, doi: 10.1093/humupd/dms015, indexed in Pubmed: 22523109.

15. Lee CC, Jan HJ, Lai JH, et al. Nodal promotes growth and invasion in human gliomas. Oncogene. 2010; 29(21): 3110-3123, doi: 10.1038/onc.2010.55, indexed in Pubmed: 20383200. 